\title{
A Method of Measuring the Solubilities of Hydrocarbons in Aqueous Solutions*
}

\author{
Robert L. Brown and Stanley P. Wasik \\ Institute for Materials Research, National Bureau of Standards, Washington, DC 20234
}

(December 7, 1973)

\begin{abstract}
An apparatus is described which measures the equilibrium distribution of a hydrocarbon between a gas phase and a liquid water phase. The method involves a multiple equilibration procedure which requires the analysis of only the gas phase. Gas-liquid chromatography was used for the hydrocarbon analysis because of its high sensitivity and selectivity. Supplemented by vapor pressure data, the observed distribution can be used to calculate the solubility of the hydrocarbon in the liquid phase. This was done for benzene, toluene, and ethylbenzene in distilled water over the temperature range 5 to $20{ }^{\circ} \mathrm{C}$ and in an artificial seawater over the temperature range 0 to $20{ }^{\circ} \mathrm{C}$. The various factors affecting the accuracy of the results are discussed in detail.
\end{abstract}

Key words: Aqueous solutions; benzene solubility; hydrocarbons; partition coefficients; seawater.

\section{Introduction}

A knowledge of the equilibrium properties of aqueous hydrocarbon solutions is valuable in several fields. In water pollution control such information is helpful in devising abatement processes [1], ${ }^{1}$ in modeling natural water systems [2], in designing toxicity experiments, and in developing analytical techniques. In petroleum research it is useful for understanding how hydrocarbons migrate and accumulate to form oil fields [3]. In biology a knowledge of how hydrocarbons behave in water is important for understanding the effects of hydration on the configuration of biopolymers [4]. And in chemistry, experimental data on these solutions are needed for testing models of water and aqueous solutions [5].

These aqueous solutions can be characterized by determining the concentration of a particular hydrocarbon. in both the solution and in the vapor above the solution. The ratio of its concentration in the two phases is an equilibrium constant called the partition coefficient. The solubility of a hydrocarbon is the maximum concentration it can have at equilibrium in the solvent at a given temperature and can be determined from the values of the partition coefficient and the vapor pressure of the pure liquid hydrocarbon at that temperature.

Although there have been many measurements of hydrocarbon solubilities in water, most have been done at only one temperature. Furthermore, there have been very few measurements on hydrocarbons in seawater. Because of the increasing need for informa-

\footnotetext{
* This work was supported by the Office of Marine Technology, Maritime Administration.

' Figures in brackets indicate the literature references at the end of this paper.
}

tion about these systems we have undertaken a program of measuring partition coefficients and their temperature dependence for a variety of hydrocarbons in aqueous solution. Our method involves a procedure which requires analysis of only the vapor phase. The analytical technique used is called headspace gas analysis [6]. This is widely used for determining the volatile constituents of biological fluids. We used gasliquid chromatography for the hydrocarbon analysis because of its high sensitivity and selectivity for these compounds. The hydrocarbons are introduced into the apparatus as vapors to avoid the danger of emulsion formation [7], which arises when liquid hydrocarbons are mixed with water. In this report we describe the apparatus and discuss the various factors affecting the precision and accuracy of the partition coefficients.

The apparatus in its present configuration is best suited for measuring partition coefficients whose values lie in the range 1 to 10 . The partition coefficients of many aromatic hydrocarbons fall in this range. Our method has a number of advantages over the spectrophotometric techniques that have been widely employed in the past for solubility measurements on these compounds. Because of the selectivity of the chromatographic analysis, compound purity is not a critical factor. This selectivity also means that several compounds can be studied at the same time. To accomplish this, high sensitivity is also necessary. A hydrogen flame detector was used and its high sensitivity for hydrocarbons made it possible to work at low concentrations where interactions between compounds are negligible. The spectrophotometric method is limited to compounds having high solubilities or large absorption coefficients. On the other hand, the high 
sensitivity of the flame detector allows one to study a much larger number of compounds. Furthermore, absolute calibration of the detector is not necessary. Only its linearity need be established and the apparatus itself can be used to do this.

To test the method, we measured the solubilities of benzene, toluene, and ethylbenzene in distilled water. These compounds have been examined extensively by several investigators using ultraviolet absorption spectroscopy to measure the hydrocarbon concentration in the liquid phase. Because of their relatively high solubilities and toxicity to marine organisms [8], these hydrocarbons may be significant factors in the oil spill problem. For this reason we also used this apparatus to measure their solubilities in seawater. Partition coefficients for the compounds in distilled water were measured over the temperature range 5 to $20{ }^{\circ} \mathrm{C}$. The partition coefficients in an artificial seawater were measured over the temperature range 0 to $20^{\circ} \mathrm{C}$. Solubilities were then determined from the partition coefficients and vapor pressure data.

\section{Description of the Apparatus and Experiments}

\subsection{Principle of the Experimental Methods Used}

In this section we give a simplified analysis of the experimental procedures. A detailed analysis of how the partition coefficients were evaluated from the observed relative hydrocarbon concentrations is given in the Appendix.

\section{Determination of Absolute Partition Coefficients}

The equilibrium distribution of a solute between two phases can be determined by measuring its relative concentration in only one phase [9]. Consider a system composed of (1) a gas phase and (2) a liquid phase, having volumes $V_{1}$ and $V_{2}$, into which we introduce some hydrocarbon solute molecules. (Our particular system contains a gas phase consisting of helium with a little water and hydrocarbon vapor, and a liquid phase consisting of distilled water or seawater and a small amount of dissolved helium and hydrocarbon.) Let the equilibrium concentrations of the hydrocarbon solute in the gaseous and liquid phases be $C_{1}$ and $C_{2}$, respectively, and let the partition coefficient $K$ be defined as the ratio $C_{2} / C_{1}$. We assume that the solute concentration is sufficiently low in each phase so that there will be no interactions between solute molecules; i.e., $K$ will not depend on $C_{1}$ and $C_{2}$. The total number of moles $N$ of solute in the system at equilibrium is $C_{1}\left(\mathrm{~V}_{1}+K V_{2}\right)$. Suppose we now remove a fraction $\alpha$ of the solute molecules from the gas phase and allow the system to equilibrate again. (In our system, the hydrocarbon molecules are removed simply by discarding a known portion of the gas phase and replacing it with pure helium.) Let $C_{1}^{\prime}$ be the gas phase concentration of the solute at equilibrium after the extraction of $\alpha C_{1} V_{1}$ moles of solute. The total number of moles of solute in the system is now
$N^{\prime}=C_{1}^{\prime}\left(V_{1}+K V_{2}\right)=N-\alpha C_{1} V_{1}=C_{1}\left[(1-\alpha) V_{1}+K V_{2}\right]$.

We define the extraction factor $F$ by

$$
F \equiv C_{1}^{\prime} / C_{1}
$$

From eq (1), we see that the gas phase concentration ratio $F$ is related to $K$ by the expression

$$
K=\left(V_{1} / V_{2}\right)(F+\alpha-1)(1-F)^{-1} .
$$

Using this procedure, one can determine $K$ from a knowledge of the volumes of the phases and the observed relative solute concentrations in one phase. We shall refer to this procedure as an extraction experiment.

\section{b. Partition Coefficients at Different Temperatures}

The temperature dependence of the partition coefficients can be determined by doing extraction experiments at different temperatures. It is possible, however, without removing any of the hydrocarbon from the system, to obtain the partition coefficient at various temperatures in terms of its value at some reference temperature $T_{R}$. This can be done simply by measuring the relative hydrocarbon concentration in the gas phase as the temperature of the system is changed. Consider the system described in the preceding paragraph. Suppose the total number of moles of solute in the system at equilibrium is $N=C_{1}\left(V_{1}+\right.$ $\left.K V_{2}\right)$. As the temperature of the liquid phase changes, $N$ remains constant, and the volumes $V_{1}$ and $V_{2}$ also remain approximately constant. Let $K$ and $K_{R}$ be the values of the partition coefficient, and $C_{1}$ and $C_{1 R}$ be the corresponding solute concentrations in the gas phase at the liquid phase temperatures $T$ and $T_{R}$, respectively. Also, we define a concentration ratio $G$,

$$
G \equiv C_{1} / C_{1 R}
$$

Then $K$ can be expressed in terms of $G$ and $K_{R}$;

$$
K=\left(V_{1} / V_{2}\right)(1-G) G^{-1}+K_{R} / G .
$$

This procedure will be referred to as a nonextraction experiment.

\subsection{Apparatus}

A schematic diagram of our apparatus is shown in figure 1. It consists of two major parts:

(1) A glass equilibration cell of volume $V_{0}\left(\approx 75 \mathrm{~cm}^{3}\right)$, which contains a volume $V_{1}\left(\approx 45 \mathrm{~cm}^{3}\right)$ of the aqueous solution. It also contains a small amount of the gas phase of volume $V_{a}=V_{0}-V_{L}$.

(2) A gas container of volume $V_{p}\left(\approx 160 \mathrm{~cm}^{3}\right)$, also made of glass. The gas in this container is that portion of the gas phase which is removed and replaced with pure helium during an experiment.

These two containers are connected by a system of 


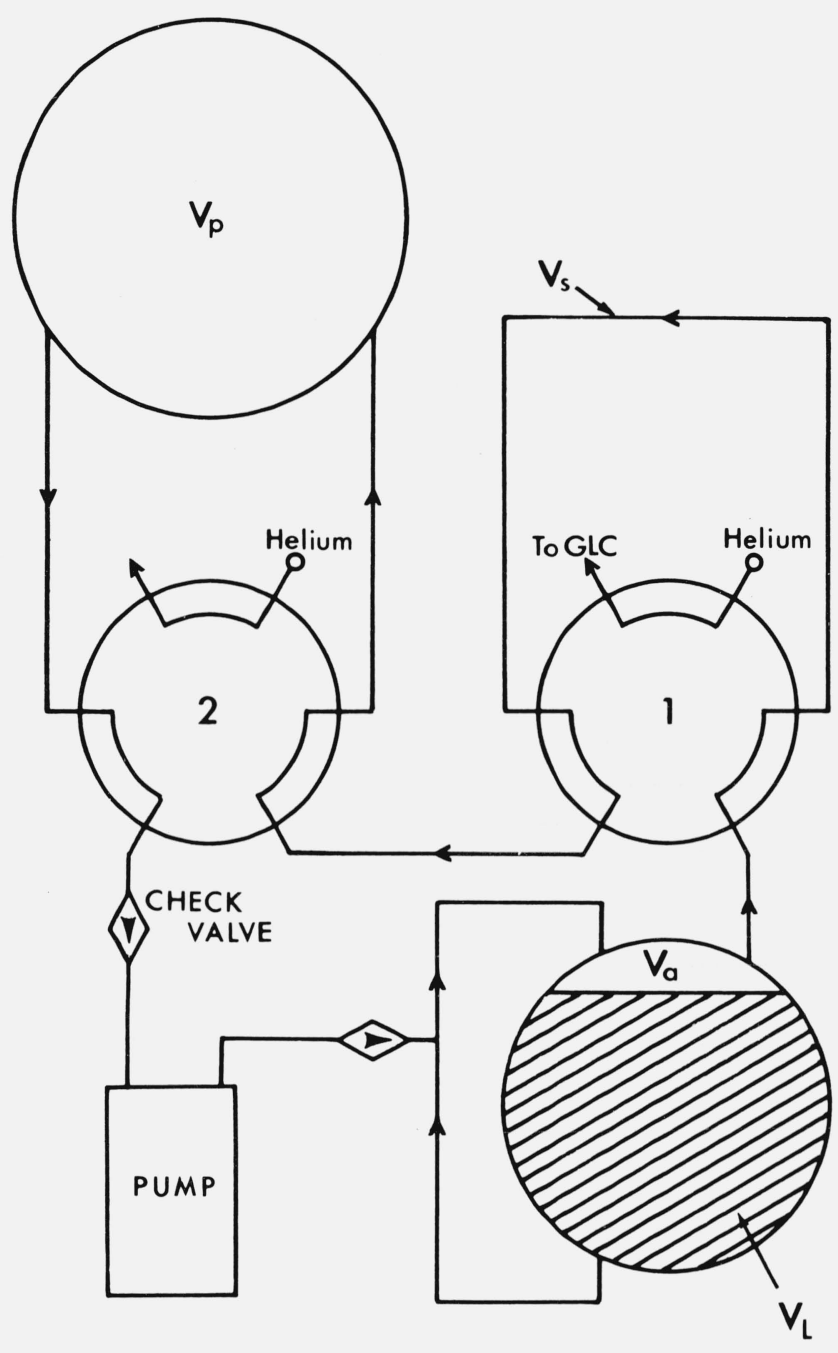

FiguRE 1. Schematic diagram of the apparatus.

The total volume of the present apparatus is about $265 \mathrm{~cm}^{3} ; V_{p} \approx 160 \mathrm{~cm}^{3}$ and $V_{L} \approx 45 \mathrm{~cm}^{3}$ The remaining volume is about $60 \mathrm{~cm}^{3}$; this arises almost entirely from the volume of gas in the equilibration cell and from the volume in the pump.

stainless-steel tubing through which helium carrier gas circulates. The volume of the connecting tubing is considerably smaller than the combined volume of the equilibration cell and the gas volume $V_{p}$.

Helium carrier gas is continuously pumped from the gas container containing volume $V_{p}$, through the equilibration cell, and then returned to $V_{p}$. The pump is a metal bellows having a displacement of $2 \mathrm{~cm}^{3}$ and was built by the NBS shops. It runs at about 100 strokes per minute. The helium enters the equilibration cell through a liquid-filled side-arm. It enters the side-arm below the surface of the liquid, and as it rises, moves liquid from the cell up the side-arm and back into the cell. In the cell, a liquid surface area of approximately $40 \mathrm{~cm}^{2}$ is exposed to the gas phase. At the pumping rates used, a liquid volume equal to the total volume of liquid in the cell circulates through the side-arm every $1.5 \mathrm{~min}$. This arrangement has the advantage that a single pump circulates both gas and liquid.
After leaving the equilibration cell, the helium passes through the first of two 6-port gas sampling valves. By switching this valve, a small sample $\left(V_{s} \approx 0.25 \mathrm{~cm}^{3}\right)$ of the hydrocarbon-containing carrier gas can be sent to the chromatograph for analysis without interrupting the circulation. The carrier then passes to the second valve and through $V_{p}$. Switching this second valve allows the gas in $V_{p}$ to be purged and replaced with pure helium at atmospheric pressure. From $V_{p}$ the gas returns to the pump. It completes one circuit through the apparatus in slightly less than a minute.

Before assembly, the metal portions of the apparatus were cleaned with trichloroethylene; the glass parts were washed with 10 percent by weight aqueous $\mathrm{HF}$ and rinsed several times with distilled water.

The equilibration cell is contained in a water bath whose temperature is controlled to within $0.01{ }^{\circ} \mathrm{C}$. The rest of the system is enclosed by an air bath held at $100 \pm 0.05{ }^{\circ} \mathrm{C}$.

The chromatographic column was a $1 / 8$-in by 6 - $\mathrm{ft}$ piece of stainless-steel tubing packed with 10 percent w/w silicone gum rubber (methyl vinyl) on Chromosorb W-AW-DMCS 80-100 mesh, kept at $100{ }^{\circ} \mathrm{C}$. Peak areas were measured with an electronic integrator.

The helium used for the carrier gas had a purity of 99.999 volume percent. The benzene, toluene, and ethylbenzene were of 99.99 mole percent purity. Distilled water from the laboratory supply was used without any purification except prolonged extraction with the helium carrier. The sea water was artificial. It was prepared according to a recipe given by Sverdrup et al. [10], and had a chlorinity value of $19.00 \%$. (The chlorinity is the total amount of chlorine, bromine, and iodine in grams contained in $1 \mathrm{~kg}$ of seawater assuming that the bromine and the iodine have been replaced by chlorine. [10])

\subsection{Experimental Procedures}

To begin a determination of the absolute partition coefficients by the extraction procedure, the entire apparatus including the liquid was first purged with helium. Then, a gaseous mixture containing benzene, toluene, and ethylbenzene was introduced into the apparatus. This was done by putting value number two (see fig. 1) in the purge position and replacing the pure helium in $V_{p}$ with helium which had passed through a saturator containing a mixture of liquid hydrocarbons. This valve was then switched back to the position shown in figure 1 , and the gas mixture was circulated through the apparatus and allowed to equilibrate for at least $1 / 2 \mathrm{~h}$ with the liquid phase. The pressures of the hydrocarbons initially in $V_{p}$ were adjusted so that their pressures in the circulating carrier gas were always considerably below their vapor pressures at the temperature of the equilibration cell. Periodic measurements of the relative hydrocarbon concentrations in the circulating carrier showed no further change $10 \mathrm{~min}$ after their introduction. The equilibration time was thus quite short. After the first equilibration, the hydrocarbon molecules in $V_{p}$ were removed by switching $V_{p}$ out of the circulating part of the 
apparatus and purging it with pure helium. $V_{p}$ was then switched back to the circulating part of the apparatus and the gas in it was allowed to equilibrate a second time with the liquid phase.

An extraction experiment was normally followed by the nonextraction procedure. This was done by varying the temperature of the equilibration cell while measuring the gas phase solute concentrations. After each temperature change, $45 \mathrm{~min}$ was allowed for the phases to equilibrate.

\section{Results}

We have described two types of experiments:

(1) The extraction procedure utilizes the concentration ratio $F$. This is the ratio of the equilibrium gas phase concentration of a particular hydrocarbon before and after extraction of a known fraction of the gas phase. The absolute value of the partition coefficient $K$ is obtained from $F$ through use of eq (3). (This is analogous to eq (A2) in the appendix.) These $K$ values become the reference values $K_{R}$ for use in the nonextraction procedure.

(2) The nonextraction procedure utilizes the concentration ratio $G$ and combines this with a reference $K_{R}$ determined from an extraction experiment. The quantity $G$ is the ratio of the observed gas phase concentration of a particular hydrocarbon at the temperature at which $K_{R}$ was determined, to its concentration observed at some other temperature. In this procedure, no gas is removed from the apparatus. Absolute $K$ values are determined from $K_{R}$ and $G$ through use of eq (5) (eq (A3), appendix).

Our observed values of the partition coefficients of benzene, toluene, and ethylbenzene in distilled water water are given in table 1. Absolute values were determined from extraction experiments performed at two temperatures, 11.75 and $20.06{ }^{\circ} \mathrm{C}$. The values shown are averages of several determinations at each temperature. Partition coefficients at the other temperatures listed in the table were obtained from nonextraction experiments. For each compound, the value at $7.06^{\circ} \mathrm{C}$ was determined using the average value of $K$ at
$11.75{ }^{\circ} \mathrm{C}$ as the reference partition coefficient $K_{R}$. The values at the remaining temperatures were determined using the average value of $K$ at $20.06{ }^{\circ} \mathrm{C}$ as the reference partition coefficient.

Next to each $K$ value listed in table 1, there are two numbers in parentheses. The first is the standard deviation of the $K$ values determined from the nonextraction experiments. It reflects the dispersion observed in the concentration ratios $G$. It gives an estimate of the dispersion encountered in determining $K$ values relative to the appropriate reference values $K_{R}$. The second number in parentheses is the standard deviation for the absolute $K$ values. For $K$ values determined by the extraction procedure it is simply the observed standard deviation of the $K$ values determined from the ratio $F$ through use of eq (3) (eq (A2)). It reflects the dispersion in the ratio $F$. The standard deviation for the absolute $K$ values determined from the nonextraction experiments and the $K_{R}$ values was calculated by combining the observed standard deviations of $G$ and $K_{R}$ through the use of eq (5) (eq (A3)). Formulas for the calculation of error propogation were obtained from reference [11].

The main cause of the dispersion in the $K$ values arises from the dispersion in the relative concentration measurements. This, in turn, arises for the most part from the variation in sample size caused by the pressure pulsations in the sample loop $V_{s}$ produced by the action of the pump. Separate experiments were conducted to estimate the dispersion involved in making the relative concentration measurements. Let us define an observation as one measurement of the relative concentration of one particular component in one sample of volume $V_{s}$ of the circulating gas. Repeated observations are considered to correspond to repeated injections of samples of the circulating gas into the chromatograph under the same experimental conditions. (A small portion of the gas, $\approx 0.1 \%$, is removed from the apparatus each time a sample is taken, and the observations are corrected for this.) A group of 60 observations of relative benzene concentrations was found to have a coefficient of variation of 0.75 percent. For gas samples taken at constant pressure,

TABLE 1. Averaged values of determined partition coefficients of benzene, toluene, and ethylbenzene in distilled water

\begin{tabular}{|c|c|c|c|c|c|c|c|c|c|}
\hline \multirow{2}{*}{ Temp. $\left({ }^{\circ} \mathrm{C}\right)$} & \multirow{2}{*}{$\begin{array}{l}\text { Number of } \\
\text { observations }\end{array}$} & \multicolumn{6}{|c|}{ Partition coefficients $K$} & \multirow{2}{*}{\multicolumn{2}{|c|}{$\begin{array}{c}\text { Predicted deviations } \\
\text { standard }\end{array}$}} \\
\hline & & \multicolumn{2}{|c|}{ Benzene } & \multicolumn{2}{|c|}{ Toluene } & \multicolumn{2}{|c|}{ Ethylbenzene } & & \\
\hline 4.50 & 3 & $12.33(0.14)^{\mathrm{a}}$ & $(0.24)^{\mathrm{b}}$ & $12.92(0.13)^{\mathrm{a}}$ & $(0.23)^{b}$ & $12.31(0.12)^{\mathrm{a}}$ & $(0.23)^{b}$ & $(0.13)^{\mathrm{a}}$ & $(0.22)^{\prime}$ \\
\hline 6.33 & 3 & $11.10(0.04)$ & $(0.18)$ & $11.42(0.04)$ & $(0.18)$ & $10.70(0.04)$ & $(0.18)$ & $(0.12)$ & $(0.20)$ \\
\hline 7.06 & 3 & $10.45(0.11)$ & $(0.19)$ & $10.68(0.10)$ & $(0.18)$ & $9.91(0.08)$ & $(0.17)$ & $(0.12)$ & $(0.32)$ \\
\hline 8.96 & 3 & $9.52(0.11)$ & $(0.19)$ & $9.61(0.11)$ & $(0.19)$ & $8.82(0.09)$ & $(0.18)$ & $(0.11)$ & $(0.18)$ \\
\hline $11.75^{\mathrm{c}}$ & 6 & $8.17(0.00)$ & $(0.10)$ & $8.05(0.00)$ & $(0.10)$ & $7.20(0.00)$ & $(0.09)$ & $(0.00)$ & $(0.19)$ \\
\hline 12.10 & 3 & $8.03(0.07)$ & $(0.16)$ & $7.92(0.07)$ & $(0.16)$ & $7.11(0.06)$ & $(0.15)$ & $(0.10)$ & $(0.16)$ \\
\hline 15.10 & 3 & $6.91(0.09)$ & $(0.16)$ & $6.68(0.09)$ & $(0.16)$ & $5.85(0.08)$ & $(0.15)$ & $(0.09)$ & $(0.15)$ \\
\hline 17.93 & 3 & $6.18(0.04)$ & $(0.12)$ & $5.85(0.04)$ & $(0.12)$ & $5.04(0.04)$ & $(0.12)$ & $(0.08)$ & $(0.13)$ \\
\hline $20.06^{\mathrm{c}}$ & 4 & $5.51(0.00)$ & $(0.11)$ & $5.14(0.00)$ & $(0.11)$ & $4.36(0.00)$ & $(0.12)$ & $(0.00)$ & $(0.10)$ \\
\hline
\end{tabular}

a This number is the standard deviation of the $K$ values determined from the nonextraction experiments. It is an estimate of the dispersion found in determining $K$ values relative to the appropriate reference values $K_{R}$.

b This number is the standard deviation for the absolute $K$ values.

c The value of $K$ determined at this temperature was used as the reference value $K_{R}$.

${ }^{\mathrm{d}}$ From propogation of error formula (p. 459 in text). 
TABLE 2. Averaged values of determined partition coefficients of benzene, toluene, and ethylbenzene in artificial seawater

\begin{tabular}{|c|c|c|c|c|c|c|c|c|c|}
\hline \multirow{3}{*}{$\begin{array}{c}\text { Temp. } \\
0.19\end{array}$} & \multirow{3}{*}{$\begin{array}{c}\begin{array}{c}\text { Number of } \\
\text { observations }\end{array} \\
3\end{array}$} & \multicolumn{6}{|c|}{ Partition coefficients $K$} & \multirow{2}{*}{\multicolumn{2}{|c|}{$\begin{array}{c}\text { Predicted standard } \\
\text { deviations }\end{array}$}} \\
\hline & & \multicolumn{2}{|c|}{ Benzene } & \multicolumn{2}{|c|}{ Toluene } & \multicolumn{2}{|c|}{ Ethylbenzene } & & \\
\hline & & $12.17(0.18)^{\mathrm{a}}$ & $(0.25)^{\mathrm{b}}$ & $12.58(0.18)^{\mathrm{a}}$ & $(0.25)^{\mathrm{b}}$ & $12.12(0.17)^{\mathrm{a}}$ & $(0.24)^{\mathrm{b}}$ & $(0.11)^{\mathrm{a}}$ & $(0.16)^{b}$ \\
\hline 5.32 & 3 & $8.96(0.08)$ & $(0.16)$ & $8.88(0.07)$ & $(0.15)$ & $8.16(0.06)$ & $(0.15)$ & $(0.10)$ & $(0.14)$ \\
\hline 10.05 & 3 & $6.91(0.12)$ & $(0.17)$ & $6.59(0.12)$ & $(0.17)$ & $5.83(0.11)$ & $(0.16)$ & $(0.09)$ & $(0.12)$ \\
\hline 14.96 & 3 & $5.35(0.06)$ & $(0.11)$ & $4.92(0.06)$ & $(0.11)$ & $4.19(0.06)$ & $(0.11)$ & $(0.07)$ & $(0.10)$ \\
\hline $20.04^{\mathrm{c}}$ & 3 & $4.18(0.00)$ & $(0.11)$ & $3.70(0.00)$ & $(0.10)$ & $3.05(0.00)$ & $(0.10)$ & $(0.00)$ & $(0.07)$ \\
\hline
\end{tabular}

a This number is the standard deviation of the $K$ values determined from the nonextraction experiments. It is an estimate of the dispersion found in determining $K$ values relative to the appropriate reference values $K_{R}$.

b This number is the standard deviation for the absolute $K$ values.

c The value of $K$ determined at this temperature was used as the reference value $K_{R}$.

on the other hand, the coefficient of variation was only 0.15 percent. This represents the reproducibility of the detection system.

With 0.75 percent as the coefficient of variation for relative concentration measurements, it was possible to calculate the dispersions to be expected in determining $F$ and $G$, and, through eqs (3) and (5) (eqs (A2) and (A3)), in the derived $K$ values. The standard deviation determined for $K$ in this fashion is shown in the last column in table 1 . As before, the first number in parenthesis is the standard deviation of the $K$ values relative to the reference $K$ values $K_{R}$ at 11.75 and $20.06{ }^{\circ} \mathrm{C}$. The agreement of these calculated standard deviations with those determined from the measurements shown in the table is satisfactory.

We believe that the standard deviations shown in table 1 give a good estimate of the uncertainty in the absolute $K$ values. As mentioned earlier, essentially all of the error in these experiments arises from the concentration measurements. The errors involved in measuring the other parameters - (pressures, volumes, and temperatures) - in eqs (A2) and (A3) are negligible by comparison.

With regard to systematic errors, our major concern is that a significant fraction of the hydrocarbon could be adsorbed on the surface of the apparatus rather than in the gas phase. To check for such effects, the extraction factor for the dry apparatus was examined for a mixture of benzene, toluene, and ethylbenzene and found to be the same for all the compounds and also equal to the calculated value. In another experiment with the dry apparatus, the surface-to-volume ratio of the volume $V_{p}$ was increased by a factor of four. The compounds again exhibited the calculated extraction factor. These experiments indicated that surface adsorption was a negligible factor in these measurements.

Lack of detector linearity would consitute another source of systematic error. The apparatus itself can be used to check the response of the detector. If there is no liquid water in the apparatus, each time the gas in $V_{p}$ is removed and replaced with pure helium, a constant, known fraction of the hydrocarbon is removed from the apparbtus. The linearity of the detector for benzene, toluene, and ethylbenzene was confirmed over a 100 -fold concentration range by repeatedly extracting the gas in $V_{p}$. Two such experi-
TABLE 3. Solubility of benzene in distilled water

\begin{tabular}{|c|c|c|c|}
\hline \multirow{2}{*}{ Temp. $\left({ }^{\circ} \mathrm{C}\right)$} & \multicolumn{2}{|c|}{ Solubility $S$ (wt. percent) } & \multirow{2}{*}{ Ref. [14] } \\
\hline & Present work & Ref. [13] & \\
\hline $\begin{array}{r}4.5 \\
4.9 \\
5.0 \\
5.2 \\
6.3 \\
6.7 \\
7.1 \\
9.0 \\
10.0 \\
11.8 \\
12.1 \\
12.5 \\
14.9 \\
15.0 \\
15.1 \\
17.9 \\
20.0 \\
20.1 \\
20.6 \\
21.0 \\
24.8 \\
24.9 \\
25.6\end{array}$ & $\begin{array}{c}0.184(0.0033)^{\mathrm{a}} \\
.185(0.0033) \\
.181(0.0055) \\
.181(0.0034) \\
.177(0.0041) \\
.177(0.0035)\end{array}$ & $\begin{array}{l}.172(0.0002) \\
.173(0.0026) \\
.171(0.0021) \\
.172(0.0020) \\
.171(0.0034) \\
.174(0.0024)\end{array}$ & $\begin{array}{l}.179 . \\
.179(0.0002) \text {. }\end{array}$ \\
\hline \multicolumn{4}{|c|}{ Other values } \\
\hline $\begin{array}{r}9.8 \\
10.0 \\
15.0 \\
17.0 \\
20.0 \\
24.0 \\
25.0\end{array}$ & $\begin{array}{l}0.176 \text { Ref. [15]. } \\
.175 \text { Ref. [16]. } \\
.150 \text { Ref. [17]. } \\
.171(0.0008) \mathrm{R} \\
.139 \text { Ref. [19]. } \\
.172(0.0008) \mathrm{R} \\
.140 \text { Ref. [20]; } \\
\text { Ref. [16]; 0.17 } \\
0.178(0.0045) \\
\text { Ref. [25]. }\end{array}$ & $\begin{array}{l}\text { [18]. } \\
178 \text { Ref. }[15] ; 0.1 \\
\text { (0.001) Ref. [21 } \\
\text { ef. [23]; } 0.176(0 .\end{array}$ & $\begin{array}{l} \\
\\
30 \text { Ref. [19]; } 0.186 \\
0.189 \text { Ref. [22]; } \\
\text { 3) Ref. [24]; } 0.174\end{array}$ \\
\hline
\end{tabular}

a The numbers in parentheses are standard deviations.

ments done at different air bath temperatures gave values of $F$ that were within 0.4 percent of the value calculated for the dry system.

Our results for the partition coefficients of benzene, toluene, and ethylbenzene in the artificial sea water are shown in table 2 . Absolute values were determined from extraction experiments at $20.06{ }^{\circ} \mathrm{C}$. The values at the other temperatures were determined from nonextraction experiments using the average value of 
$K$ at $20.06{ }^{\circ} \mathrm{C}$ as the reference partition coefficient $K_{R}$. The standard deviations shown in parentheses have the same meaning as in table 1.

To convert the partition coefficients to solubilities, it is necessary to use vapor pressure data. Our partition coefficients were measured at hydrocarbon pressures of the order of 1-5 percent of their vapor pressures at any particular temperature. Under such dilute conditions the activity of the hydrocarbon in either the vapor or the liquid is proportional to its concentration. We shall assume that the proportionality holds in the liquid phase for all concentrations up to a saturated solution. In the gas phase, however, a very small correction must be made for the departure of the hydrocarbon vapor from ideal gas behavior. One can show that the weight percent $S$ of a hydrocarbon in an aqueous solution in equilibrium with a liquid hydrocarbon can be written as

$$
S=\left(M / \rho_{L}\right)(p / R T) K(1+2 B p / R T)(100)
$$

where $M$ is the molecular weight of the hydrocarbon, $\rho_{L}$ is the density of the liquid solution, $K$ is the partition coefficient measured at low hydrocarbon pressures, $p$ is the vapor pressures of the hydrocarbon at the temperature $T, R$ is the gas constant, and $B$ is the second virial coefficient for hydrocarbon-hydrocarbon interactions. When liquid hydrocarbon is present at equilibrium, it will dissolve some water. The amount dissolved is so small, however, that no correction was made for its effect on the hydrocarbon vapor pressures [12]. Also, no correction was made for the contribution of hydrocarbon-water interactions to the second virial coefficients.

Our results for the solubility of benzene at different temperatures in distilled water are given in table 3 along with literature values. When available, standard deviations are listed in parentheses after each $S$ value. In this temperature region there have been only two other extensive sets of measurements; those of Arnold et al. [13], and those of Bohon and Clussen [14]. Both groups used spectrophotometric analytical techniques. Our results agree satisfactorily with those of Bohon and Claussen over the whole temperature range, but are systematically higher than those of Arnold et al., by about 4 percent over most of the range.

Our results for the solubilities of toluene and ethylbenzene in distilled water are shown in tables
TABLE 4. Solubility of toluene in distilled water

\begin{tabular}{|c|c|c|}
\hline \multirow{2}{*}{ Temp. $\left({ }^{\circ} \mathrm{C}\right)$} & \multicolumn{2}{|c|}{ Solubility $S$ (wt. percent) } \\
\hline & Present work & Ref. [14] \\
\hline $\begin{array}{r}0.4 \\
3.6 \\
4.5 \\
6.3 \\
7.1 \\
9.0 \\
10.0 \\
11.2 \\
11.8 \\
12.1 \\
14.9 \\
15.1 \\
15.9 \\
17.9 \\
20.1 \\
25.6\end{array}$ & $\begin{array}{c}0.0612(0.0010)^{\mathrm{a}} \\
.0601(0.0011) \\
.0586(0.0018) \\
.0587(0.0011) \\
\\
.0573(0.0014) \\
.0575(0.0012) \\
.0569(0.0013) \\
.0577(0.0013) \\
.0566(0.0011)\end{array}$ & $\begin{array}{l}.0628 \\
.0624(0.0000) \\
.0622(0.0003) \\
.0620(0.0000) \\
.0623(0.0004)\end{array}$ \\
\hline \multicolumn{3}{|c|}{ Other values } \\
\hline $\begin{array}{l}16.0 \\
25.0\end{array}$ & \multicolumn{2}{|c|}{$\begin{array}{l}0.047 \text { Ref. [26] } \\
.0536(0.0003) \text { Ref. [21]; } 0.053 \text { Ref. [25]; } \\
.0515(0.0017) \text { Ref. }[23]\end{array}$} \\
\hline
\end{tabular}

a The numbers in parentheses are standard deviations.

TABLE 5. Solubility of ethylbenzene in distilled water

\begin{tabular}{c|c|c}
\hline \hline \multirow{2}{*}{ Temp. $\left({ }^{\circ} \mathrm{C}\right)$} & \multicolumn{2}{|c}{ Solubility $S$ (wt. percent) } \\
\cline { 2 - 3 } & Present work & Ref. [14] \\
\hline 0.4 & & $0.0219(0.0003)$ \\
4.5 & $0.0196(0.0004)^{\mathrm{a}}$ & \\
5.2 & & $.0213(0.0001)$ \\
6.3 & $.0192(0.0004)$ & \\
7.1 & $.0186(0.0006)$ & \\
9.0 & $.0187(0.0004)$ & \\
11.8 & $.0181(0.0005)$ & \\
12.1 & $.0183(0.0004)$ & \\
15.1 & $.0180(0.0005)$ & \\
17.9 & $.0184(0.0005)$ & .0207 \\
20.1 & $.018 ;(0.0004)$ & .0207 \\
20.7 & & $.0208(0.0002)$ \\
21.2 & & \\
25.6 & & \\
\hline
\end{tabular}

Other values

\begin{tabular}{l|l}
15.0 & 0.014 Ref [26] \\
25.0 & $\begin{array}{l}.0165(0.0002) \text { Ref. [21]; 0.0168 Ref. [27]; } \\
.0152(0.0008) \text { Ref. [23]. }\end{array}$
\end{tabular}

a The numbers in parenthesis. are standard deviations.

TABLE 6. Solubilities of benzene, toluene, and ethylbenzene in an artificial seawater having a chlorinity ${ }^{\mathrm{a}}$ value of 19.00 percent

\begin{tabular}{c|c|c|c|c}
\hline \hline \multirow{2}{*}{ Temp. } & \multirow{2}{*}{$\begin{array}{c}\text { Number of } \\
\text { observations }\end{array}$} & \multicolumn{3}{|c}{ Solubility $S$ (wt. percent) } \\
\cline { 3 - 5 } & & Benzene & Toluene & Ethylbenzene \\
\cline { 3 - 5 } & 4 & $0.1323(0.0017)^{\mathrm{b}}$ & $0.0449(0.0006)^{\mathrm{b}}$ & $0.0140(0.0002)^{\mathrm{b}}$ \\
0.19 & 4 & $.1376(0.0022)$ & $.0429(0.0007)$ & $.0133(0.0003)$ \\
5.32 & 4 & $.1347(0.0023)$ & $.0416(0.0008)$ & $.0129(0.0003)$ \\
10.05 & 4 & $.1318(0.0025)$ & $.0405(0.0008)$ & $.0125(0.0003)$ \\
14.96 & 4 & $.1296(0.0022)$ & $.0397(0.0008)$ & $.0122(0.0003)$ \\
20.04 & &
\end{tabular}

${ }^{\text {a }}$ See Ref. [10] for definition of chlorinity.

b The numbers in parentheses are standard deviations for the absolute $K$ values. 
4,5 , and 6 , respectively. For these compounds, the only extensive data in our temperature range are those of Bohon and Claussen [14]; our results are systematically lower than theirs by roughly 8 percent over the whole temperature range.

\section{Appendix: Detailed Analysis of the Apparatus and Experimental procedures}

\subsection{Determination of Absolute Partition Coefficients}

The area under the chromatographic peak is proportional to the hydrocarbon concentration in the sample volume $V_{s}$ (see fig. 1). One must relate measurements of peak areas after successive equilibrations to the partition coefficient. The general way this is done was outlined in section 2.1a. In the detailed analysis we take into account the fact that different parts of the apparatus are at different temperatures, and also different pressures because of the viscous drag on the circulating carrier gas. For completeness we also consider effects arising from nonideal gas behavior even though they happen to be too small to be important in the measurements on benzene, toluene, and ethylbenzene. (They are important, however, in relating the partition coefficients to solubilities.)

The analysis is based on the assumption that thermal diffusion effects will be negligible because of the thorough mixing resulting from the carrier circulation. Thus, the composition of the gas phase will be the same in all parts of the apparatus. It is convenient to divide the gaseous portion of the apparatus into several parts which may differ in temperature and total pressure. Let $V_{i}$ be the volume of the $i$ th part, and $T_{i}$ and $P_{i}$ be its temperature and pressure. Let $C_{\alpha i}$ be the concentration of the component $\alpha$ in the volume $V_{i} . X_{\alpha}=C_{\alpha i} \bar{V}_{i}$ is the mole fraction of $\alpha$ in the mixture, and $\bar{V}_{i}$ is the volume of $1 \mathrm{~mol}$ of the mixture at the temperature and pressure $T_{i}$ and $P_{i}$. The mole fraction $x_{\alpha}$ is assumed to be the same for all the $V_{i}$. Let us define a factor $R_{i j}=C_{\alpha i} / C_{\alpha j}$. This is the ratio of the steadystate concentration of $\alpha$ in $V_{i}$ to its concentration in $V_{j}$. The volume $\bar{V}_{i}$ can be expressed in terms of the virial equation of state [28],

$$
\bar{V}_{i}=R T_{i} / P_{i}+B_{i}
$$

where $R$ is the gas constant, and $B_{i}$ is the second virial coefficient for the mixture in the volume $V_{i}$; it is a function of the temperature and composition of the mixture. Since the product $C_{\alpha i} \bar{V}_{i}$ is the same for all $V_{i}$, one has the relation

$$
R_{i j}=\left(P_{i} T_{j}\right)\left(P_{j} T_{i}\right)^{-1}\left[1+\left(P_{j} B_{j} / T_{j}-P_{i} B_{i} / T_{i}\right) / R\right] .
$$

Only terms to first order in the $B_{i}$ have been considered. This expression enables us to relate the concentration of $\alpha$ in different parts of the apparatus to $C_{\alpha s}$, its concentration in the sample loop $V_{s}$. Let us now consider how the partition coefficient can be gotten from measurements of the relative values of $C_{\alpha s}$.
We define an extraction factor $F_{\alpha}=C_{\alpha s}^{\prime} / C_{\alpha s}$, where $C_{\alpha s}$ is the concentration in $V_{s}$ during the first equilibration with the liquid phase; $C_{\alpha s}^{\prime}$ is the concentration after purging $V_{p}$ with pure helium and allowing a second equilibration. By the same arguments presented in section $2.1 \mathrm{a}$, we can show that

$$
\begin{array}{r}
K_{\alpha}=\left(F_{\alpha} \sum_{i} R_{i s} V_{i}-\sum_{i} R_{i s}^{\prime} V_{i}+R_{p s} V_{p}\right) \\
{\left[V_{L}\left(R_{a s}-F_{\alpha} R_{a s}^{\prime}\right)\right]^{-1}}
\end{array}
$$

where $K_{\alpha}$ is the partition coefficient, i.e., the ratio $C_{\alpha a} / C_{\alpha L} . C_{\alpha a}$ is the equilibrium concentration of $\alpha$ in the gas volume $V_{a}$ located just above the liquid in the equilibration cell and $C_{\alpha L}$ is its corresponding concentration in the liquid. $V_{L}$ is the volume of the liquid phase. The summations are over all the volumes in the gas phase portion of the apparatus. The parameter $R_{i j}^{\prime}$ is very slightly different from $R_{i j}$ because of the effect of the composition change on the virial coefficients $B_{i}$ and $B_{j}$. In our experiments the concentrations of the hydrocarbons were so low that the contribution of the $B_{i}$ to the $R_{i j}$ could be neglected. $R_{i j}^{\prime}$ could thus be set equal to $R_{i j}$. Repeated purges of $V_{p}$ under these conditions, followed by equilibration will thus produce the same fractional decrease in $C_{\alpha s}$.

The different volumes $V_{i}$ were measured in various ways. Some were determined by filling with water and weighing. Others were determined by pressurizing and measuring the volume of gas released when $V_{i}$ was opened to the atmosphere. Several cylindrical volumes were measured directly. The temperatures $T_{i}$ were measured with mercury thermometers. The various pressures $P_{i}$ in the apparatus were estimated by means of the Poiseuille equation. The average pressure drop across the pump was about 2 percent of the total pressure in the system.

\subsection{Determination of the Temperature Dependence of $K_{\alpha}$}

The temperature dependence of the partition coefficient can be determined by repeating the above extraction experiment at a different temperature. It is convenient, however, not to extract the hydrocarbon, but simply to observe its relative gas phase concentration at different equilibration cell temperatures. This temperature is the same as that of $V_{a}$, the gas volume just above the liquid in the equilibration cell. The concentration $C_{\alpha s}$ of $\alpha$ in the sample loop depends on the temperature $T_{a}$ of the equilibration cell in the following way,

$$
\begin{aligned}
C_{\alpha s}\left(T_{a}\right)=N_{\alpha} & \left\{R _ { a s } ( T _ { a } ) \left[V_{a}\left(T_{a}\right)\right.\right. \\
& \left.\left.+K_{\alpha}\left(T_{a}\right) V_{L}\left(T_{a}\right)\right]+\sum_{i \neq a} R_{i s} V_{i}\right\}^{-1} .
\end{aligned}
$$

The temperature dependences of $V_{a}$ and $V_{L}$ are very 
small and can be easily calculated. That of $R_{a s}$ is given by eq (Al). The quantity $N_{\alpha}$ is the total number of moles of the species $\alpha$ in the system and is constant. Let $T_{a R}$ be some reference temperature. We define the ratio $G_{\alpha}=G_{\alpha S}\left(T_{a}\right) / C_{\alpha S}\left(T_{a R}\right)$. One can use eq (A3) to calculate $K_{\alpha}\left(T_{a}\right)$ from the observed values of $G_{\alpha}$ and the value of $K_{\alpha}\left(T_{a R}\right)$ determined at $T_{a R}$ by an extraction experiment.

\section{References}

[1] Tsonopoulos, C. and Prausnitz, J. M., Ind. Eng. Chem. Fundam. 10,593 (1971).

[2] LeFeuvre, A. R., Water and Water Pollution Handbook, Vol. 1, L. L. Ciaccio, Ed. (Marcel Dekker, Inc., New York, 1971), p. 263.

[3] Peak, E. and Hodgson, G. W., J. Amer. Oil Chemists Soc. 4, 215 (1966).

[4] Ben-Naim, A. J. Chem. Phys. 57, 5257 (1972).

[5] Ben-Naim, A., Water and Aqueous Solutions, R. A. Horne, Ed. (Wiley, New York, 1972), p. 425.

[6] Luckey, M. J., J. Forensic Sciences 16, 120 (1971)

[7] Peake, E. and Hodgson, G. W., J. Amer. Oil Chemists Soc. 44, 696 (1967).

[8] Blumer, M., Environmental affairs 1, 54 (1971).

[9] McAuliffe, C., Chemical Technology, January 1971, (American Chemical Soc., Washington, DC), p. 46.

[10] Sverdrup, H. U., Johnson, M. W., and Fleming, R. H., The Oceans, (Prentice-Hall, Inc., Englewood Cliffs, New Jersey, 1942), p. 186, formula of Brujewicz (Subow, 1931).
[11] Wilson, E. B., Jr., An Introduction to Scientific Research, (McGraw-Hill, New York, 1952), p. 272.

[12] Black, C., Joris, G. G., and Taylor, H. S., J. Chem. Phys. 16, 537 (1948).

[13] Arnold, D. S., Plank, C. A., and Erickson, E. E., Chem. Eng. Data Ser. 3, 253 (1958).

[14] Bohon, R. L. and Claussen, W. F., J. Amer. Chem. Soc. 73, 1571 (1951).

[15] Vermillion, H. E., Ph.D. thesis, 1939, Duke University, Durham, NC.

[16] Stearns, R. S., Oppenheimer, H., Simon, E., and Harkins, W. D., J. Chem. Phys. 15, 496 (1947).

[17] Hill, A. E., J. Amer. Chem. Soc. 44, 1163 (1922).

[18] Franks, F., Gent, M., and Johnson, H. H., J. Chem. Soc. 2716 (1963).

[19] Hayashi, M., and Sasaki, T., Bull. Chem. Soc. Japan 29, 857 (1956).

[20] Booth, H. S. and Everson, H. E., Ind. Eng. Che. 40, 1491 (1948); 41, 2627 (1949).

[21] Morrison, T. J., and Billett, F., J. Chem. Soc. 3819 (1952).

[22] Horiba, S., Mem. Coll. Eng., Kyoto Imp. Univ. 1, 49 (1914).

[23] McAuliffe, C., J. Phys. Chem. 70, 1267 (1966).

[24] Brady, A. P., and Huff, H., J. Phys. Chem. 62, 644 (1958).

[25] Andrews, L. J., and Keefer, R. M., J. Am. Chem. Soc. 71, 3644 (1949).

[26] Fühner, H., Chem. Ber. 57, 510 (1924).

[27] Andrews, L. J., and Keefer, R. M., J. Am. Chem. Soc. 72, 5034 (1950).

[28] Hirschfelder, J. O., Curtiss, C. F., and Bird, R. B., Molecular Theory of Gases and Liquids (Wiley, New York, 1954).

(Paper 78A4-825) 